\title{
On Satisfying Timing and Resource Constraints in Distributed Multimedia Systems
}

\author{
Costas Mourlas and David Duce and Michael Wilson \\ Rutherford Appleton Laboratory, Chilton, Didcot, OX11 0QX, UK \\ E-mail: \{C.Mourlas,D.A.Duce,M.D.Wilson\}@rl.ac.uk
}

\begin{abstract}
In this paper ${ }^{1}$, we present a new synchronization strategy for multimedia applications executed in a distributed environment. This strategy makes the timing properties of the system and the quality of the media presentations predictable since one is able to determine analytically whether the timing requirements of each multimedia application will be met, and if not, which timing requirements willfail. The proposed synchronization protocol provides deterministic guarantees and service reliability that can't be compromised by resource contention. Thus, the application can maintain the initial quality of service $(Q o S)$ level without encountering unpredictable delays and blocking due to synchronization.
\end{abstract}

\section{Introduction}

There is currently considerable interest in developing multi-media applications in open distributed systems. This is motivated by the wide range of potential applications such as desktop conferencing, distributed multi-media information systems and video-on-demand services. However, it is clear that existing frameworks for open distributed systems do not support the particular requirements of distributed multi-media such as real-time constraints, intra/inter-media synchronization and real-time communication. There is also a lack of a suitable theory that makes the timing properties of a distributed multi-media application predictable.

Because of the layered design of multimedia systems, the granularity of synchronization is generally coarser at the application level, becoming more detailed at the lower levels of the system. For example, a user at the application level is concerned that a video segment begins and ends at specific time points whereas the system might be concerned with frame synchronization, real-time frame delivery and resource management. Our work is concentrated on systemlevel synchronization techniques providing a new resource

\footnotetext{
${ }^{1}$ This work has been carried out as part of the ERCIM Fellowship Programme
}

management strategy for multimedia applications executed in a heterogenous, distributed environment.

A significant amount of work has been carried out for making resource allocations to satisfy specific applicationlevel requirements and various scheduling schemes are available to ensure that the allocation decisions can be carried out. Various system-wide schemes have been studied to arbitrate resource allocation among contending applications. The Rialto operating system [2] was designed to support simultaneous execution of independent real-time and nonreal-time applications, meeting the real-time requirements of all those for which it is possible while providing liveness for the non-real-time programs. The RT-Mach microkernel [3] supports a processor reserve abstraction which permits threads to specify their CPU resource requirements. If admitted by the kernel, it guarantees that the requested CPU demand is available to the requestor. Q-RAM [8] and SMART [7] support applications with time constraints, and provides dynamic feedback to applications to allow them to adapt to the current load. The Lancaster QoS Architecture [1] provides extensions to existing micro-kernel environments for the support of continuous media. The QoS Broker model [6] addresses also the requirements for resource guarantees, QoS translation and admission control, so a new system architecture is proposed which provides all these issues. The Nemesis operating system is described in [4] as part of the Pegasus Project, whose goal is to support both traditional and multimedia applications. A large portion also of real-time scheduling theory deals with the important problem of the schedulability analysis and the predictability of a set of real-time applications [9].

We have to notice at this point that most of the above CPU allocation schemes are based on the restrictive assumption that the applications are independent of one another and do not have access to multiple resources simultaneously. In our approach we focus on "real" environments where a set of multimedia applications share a number of non-preemptable resources or access shared data (e.g. storage servers, live media sources etc.) which are part of a high-speed local area network (see figure 1). The proposed synchronization protocol, called the Set Based Synchronization Protocol, is 


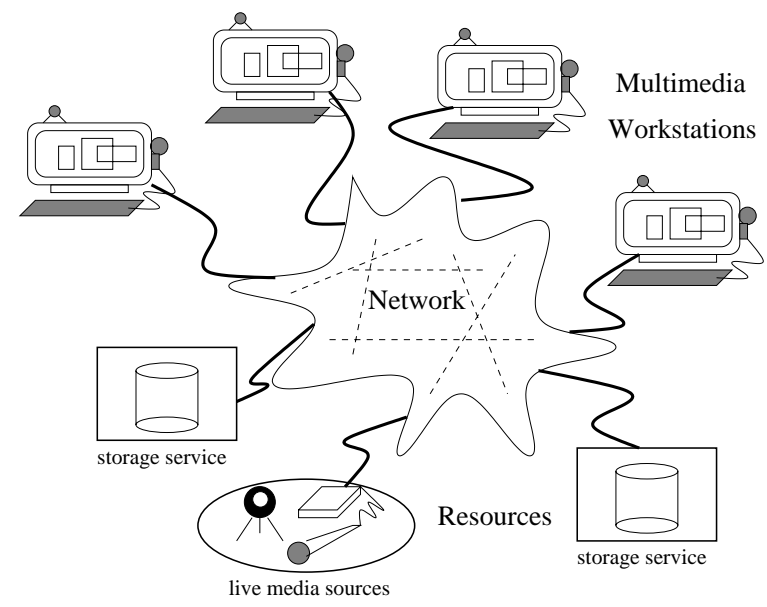

Figure 1. The distributed multimedia environment

based on the on-demand paradigm where resources are assigned only when actually required. The penalty paid for this, found also in all the on-demand approaches, is blocking. In predictable multimedia environments, the blocking has to be deterministic and for this reason our approach imposes a specific structure on blocking to bound the blocking time. The proposed protocol is an extension of our previous work [5] on distributed real-time systems.

\section{Assumptions and Notation}

In our model, we assume that the QoS for continuous media objects is expressed with temporal and spatial resolutions. The temporal resolution can be expressed by the number of frames per second ( $f p s)$ or sample rate and the spatial resolution can by expressed by data size, number of bits per pixel, compression scheme, etc. It is assumed also that every application executes periodic reads of a number of frames from a remote media server into a local buffer first and then plays them due to the fact that continuous media require periodic service activities for transmission and presentation.

In this paper, we view every different multimedia application executed in a distributed environment as a periodic task that can require in each period the use of non-preemptable resources or access shared data. For example, one multimedia session can be modelled as a task which in every $50 \mathrm{~ms}$ needs to deliver 3 video frames from the storage_server 1 and 6 audio frames from storage_server ${ }_{2}$. Since these storage servers are shared and exclusively used (i.e. guaranteed exclusive access), there is a possibility for one such task to block waiting for the use of these servers. The period of each multimedia task is determined by the desired quality of service (i.e. the temporal and spatial resolutions of the continuous media), the number of continuous media used in the application, the processor speed and the buffer size used by the node that executes the application. This means that high quality applications using many continuous media are represented by our model as tasks having short periods, i.e. high frequency tasks.

The assumptions and basic notation that will be used throughout this paper follows:

1. any continuous media application is represented by a multimedia task $\tau_{i}$ allocated on a different node $\wp_{i}$ of the distributed system and can require the use of non-preemptable resources or access shared data $R_{i}$.

2. every multimedia task $\tau_{i}$ is periodic with period $T_{i}$ and has deadline $D_{i}$ at the end of its period (i.e. $D_{i}=T_{i}$ ).

3. multimedia tasks are assigned fixed priorities inversely to their periods.

4. every task asks for all of its global resources $R_{i}$ only once in its period and subsequently can release these resources. Two operations are used for this reason:

$$
\begin{aligned}
& \text { - allocate(ResourceSet) and } \\
& \text { - release(ResourceSet). }
\end{aligned}
$$

When a task $\tau_{i}$ issues the allocate command asking for its resources it then blocks (i.e. hangs) until all these resources have been allocated to $\tau_{i}$ by the resource manager. The duration of this time interval constitutes the blocking time $B_{i}$ of the task. The section between exiting from the allocate call and the last release call constitutes the critical section of the task.

5. every multimedia task $\tau_{i}$ has known, deterministic worst-case execution time $C_{i}$. This is the total deterministic computation requirement of task $\tau_{i}$ during each period, and $C_{i}=C_{c s}^{i}+C_{n o n-c s}^{i}$ where:

$C_{c s}^{i}$ is the computation requirement of task $\tau_{i}$ within its critical section. This is the total time that $\tau_{i}$ uses the resources and the network in each period for data retrieval,

$C_{n o n-c s}^{i}$ is the deterministic computation requirement of task $\tau_{i}$ outside its critical section. This is the time in each period that $\tau_{i}$ needs to process the received data frames.

Due to the fact that every multimedia task is allocated on a different node of the distributed system, cpu scheduling is not the main problem, but since tasks are inter-dependent the main problem is task synchronization and resource allocation. Hence, blocking due to synchronization has to be deterministic in order to have nice analysis properties and a high degree of system predictability. 


\section{The Set Based Synchronization Protocol}

In this section, we present the Set Based Synchronization Protocol suitable for synchronizing multimedia tasks executing on distributed systems. A set of $n$ multimedia applications can be modelled as a set of $n$ periodic tasks $\tau_{1}, \ldots, \tau_{n}$ each one bound to a different node of the distributed system. Each task is characterized by five components $\left(C_{c s}^{i}, C_{n o n-c s}^{i}, T_{i}, D_{i}, R_{i}\right), 1 \leq i \leq n$, according to the notation and the assumptions introduced in the previous section.

In the analysis of the Set Based Synchronization Protocol, each one of the resources can be in one of the three following different states at a specific point in time during execution:

1. free if there is not any task that either asks for the use of this resource or uses this resource at this time in its critical section.

2. in use if there is a task that asked for the use of this resource for its critical section and this task is now within its critical section.

3. allocated (to a task $\tau_{j}$ ) if the task $\tau_{j}$ asked for the use of this resource, the resource has been allocated to the task $\tau_{j}$ but $\tau_{j}$ hasn't entered yet into its critical section.

Suppose that a task $\tau_{i}$ requires the use of $\kappa$ resources through a call of the form allocate $\left(R_{i}\right)$, where $R_{i}=$ $\left\{r_{1}, \ldots, r_{k}\right\}$. Then the following cases can occur:

1. All the required resources in $R_{i}$ are free. Then all these resources are allocated to the task $\tau_{i}$ and the task proceeds immediately to its critical section. The states of all resources in $R_{i}$ become in use.

2. If case 1 does not hold then the following actions are performed. If any of the $r_{1}, \ldots, r_{k}$ is free then it is allocated to $\tau_{i}$. If any of the resources in $R_{i}$ has been allocated to a lower priority task $\tau_{j}\left(T_{i}<T_{j}\right)$ and $\tau_{j}$ has not entered its critical section then it is deallocated from $\tau_{j}$ and it is allocated to $\tau_{i}$. If any of the resources in $R_{i}$ is in use then after its release it is allocated to the highest priority task that is requesting it. The task $\tau_{i}$ will proceed to its critical section if and only if all the resources in $R_{i}$ have been allocated to $\tau_{i}$.

We have to notice here that a resource is actually locked by a task only if the resource is in use by this task and not when the resource is allocated to that task. Note also that by the definition of the protocol, a task $\tau_{i}$ can be blocked by a lower priority task $\tau_{j}$, only if $\tau_{j}$ is executing within its critical section when $\tau_{i}$ requested resources and both $\tau_{i}$ and $\tau_{j}$ use common resources in their critical section, i.e. $R_{i} \cap R_{j} \neq \emptyset$.
Theorem 3.1 The Set Based Synchronization Protocol prevents deadlocks.

Proof: By definition, every task $\tau_{i}$ proceeds to its critical section if and only if all the resources in $R_{i}$ have been allocated to $\tau_{i}$. Thus, $\tau_{i}$ will never ask in its critical section for the use of any other resource and so a blocking cycle (deadlock) cannot be formed.

To perform a schedulability analysis using the proposed synchronization protocol, we define $B_{\kappa}, 1 \leq \kappa \leq n$, the longest duration of blocking that can be experienced by $\tau_{\kappa}$. Since each task is bound to a different processor, theorem 3.2 defines a sufficient set of conditions for a set of tasks to meet their deadlines.

Theorem 3.2 A set of $n$ periodic tasks, each one bound to a different processor $\wp$ can be scheduled using the Set Based Synchronization Protocol if the following conditions are satisfied:

$$
\forall i, 1 \leq i \leq n, \quad C_{i}+B_{i} \leq T_{i}
$$

Proof: The above set of inequalities state that for each task $\tau_{i}$ the sum of the blocking time $B_{i}$ and the total execution time $C_{i}$ of the task must be lower than or equal to its period $T_{i}$. If this sum which represents the completion time of $\tau_{i}$ was greater than its period and hence greater than its deadline (since $T_{i}=D_{i}$ ), task $\tau_{i}$ could not be scheduled. $\square$

Once $B_{i}$ s have been computed for all $i$, theorem 3.2 can then be used to determine the schedulability of the set of tasks.

\section{Determination of Task Blocking Time}

Here, we shall compute the worst-case blocking time that a task has to wait for its resource requirements. The fundamental objective of the Set Based Synchronization Protocol is to obtain bounded blocking times for multimedia tasks requiring the access of shared resources. The bounded waiting times in turn can be used to determine whether a set of multimedia tasks running on a distributed environment can meet their deadlines using theorem 3.2.

Assume a set of $n$ periodic tasks with $D_{i}=T_{i}$ using the Set Based Synchronization Protocol. We define as $B_{i}, 1 \leq i \leq n$, the longest duration of blocking that can be experienced by $\tau_{i}$.

Theorem 4.1 Consider a set of $n$ tasks $\tau_{1}, \ldots, \tau_{n}$ each one bound to a different processor $\wp_{i}$ and the Set Based Synchronization Protocol is used for the allocation of the resources. Let 
$H_{i}=\left\{\tau_{j} \mid T_{i}>T_{j} \wedge R_{i} \cap R_{j} \neq \emptyset\right\}$, - set of tasks having higher priorities and need common resources with $\tau_{i}$

$L_{i}=\left\{\tau_{j} \mid T_{i}<T_{j} \wedge R_{i} \cap R_{j} \neq \emptyset\right\}, \quad$ - set of tasks having lower priorities and need common resources with $\tau_{i}$

$\beta_{i}=\max \left(\left\{C_{c s}^{j} \mid \tau_{j} \in L_{i}\right\}\right) . \quad$ - blocking time due to lower priority tasks in $L_{i}$ Then, the worst case blocking time $B_{i}$ of task $\tau_{i}$ is equal to

$$
B_{i}= \begin{cases}\beta_{i}+\sum_{\forall \tau_{j} \in H_{i}} \alpha_{j}^{i} & \text { if } \sum_{\forall \tau_{j} \in H_{i}} \alpha_{j}^{i}<\min \left(\left\{T_{\kappa} \mid \tau_{\kappa} \in H_{i}\right\}\right) \\ \infty & \text { otherwise }\end{cases}
$$

where $\alpha_{j}^{i}$ is the contribution of task $\tau_{j}$ to the $B_{i}$ value and,

$\alpha_{j}^{i}= \begin{cases}0 & \text { if } \exists \tau_{l} \in H_{i}: R_{j} \cap R_{l} \neq \emptyset \wedge T_{j}<T_{l} \\ B_{j}+C_{c s}^{j} & \text { otherwise }\end{cases}$

Proof: The sum in formula 2 above represents the longest blocking time for a task $\tau_{i}$ at its worst-case task set phasing. At this worst-case phasing of the tasks, when $\tau_{i}$ wants to enter into its critical section, it finds the lower priority tasks that use common resources with $\tau_{i}$ executing within their critical sections. Then, just before all these lower priority tasks have finished their critical sections and have released their resources, all the higher priority tasks that use common resources with $\tau_{i}$ come one after the other and ask for their resources, enter their critical sections and at the end release their resources.

Thus, $B_{i}$ has two parts. The first part, namely $\beta_{i}$ is due to $L_{i}$ and it is equal to the maximum value of $C_{c s}$ among the lower priority tasks in $L_{i}$. The second part comes from tasks in $H_{i}$. In all cases, the duration of the second part should be less than the minimum period $T_{k}$ of the tasks in $H_{i}$, otherwise the task $\tau_{\kappa}$ could block repeatedly the task $\tau_{i}$ and in this case $B_{i}$ can be prohibitively large or even unbounded (condition of formula 2). This does not mean that the task set is not schedulable, rather that there is not a way to determine accurately using the proposed protocol the worst case blocking time $B_{i}$ of the task $\tau_{i}$.

Note now that the longest blocking time $B_{i}$ occurs when the blocking time of $\tau_{\lambda}, \tau_{\lambda} \in H_{i}$ does not overlap with those of other tasks in $H_{i}$ at the worst-case task set phasing for $\tau_{i}$. Thus, a task $\tau_{\lambda}$ in $H_{i}$ contributes to $B_{i}$ by $\left(B_{\lambda}+C_{c s}^{\lambda}\right)$ in case this task does not have common resources with any other task in $H_{i}$. Assume now that a task $\tau_{j}$ in $H_{i}$ has common resources with a task $\tau_{l}$ in $H_{i}$ and $\tau_{l}$ has lower priority than $\tau_{j}$. In this case, the contribution of $\tau_{j}$ to the blocking time of $\tau_{i}$ is taken care by its lower priority task $\tau_{l}$. Thus, $\tau_{j}$ may contribute indirectly to $B_{i}$ through the worst case blocking time $B_{l}$ of $\tau_{l}$. Its direct contribution is zero. As the same argument may equally apply to $\pi$, it follows that for any task $\tau_{j}$ in $H_{i}$ with common resources with others in $H_{i}$ contributes only by a factor $\left(B_{j}+C_{c s}^{j}\right)$ in case there is not any task $\tau_{k}$ with priority lower than $\tau_{j}$ sharing common resources with $\tau_{j}$. This condition is expressed by the formula 3 . Hence the Theorem follows.

As far as the case of tasks with equal periods is concerned, it is not necessary to link the priority of a task directly with its period. We can assign to each task $\tau_{i}$ a unique priority $p_{i}$ such that $\forall i, j \quad p_{i}<p_{j} \Rightarrow T_{i} \geq T_{j}$. Once these blocking terms $B_{i}, \quad 1 \leq i \leq n$, have been determined, theorem 3.2 gives a fairly complete solution for multimedia task synchronization and scheduling in the distributed environment.

\section{A Schedulability Analysis Example}

We illustrate the schedulability analysis based on the proposed synchronization protocol with the following example.

Example 5.1 Consider a distributed environment and four video presentations each one displayed on a different node of the system and represented by a task $\tau_{i}, 1 \leq i \leq 4$. This environment also supports five storage servers $r_{1}, \ldots, r_{5}$ where a collection of continuous media clips is stored.

We allocate to each task $\tau_{i}$ the minimum bandwidth Bandwidt $_{i}$ that can be provided at the worst case task phasing. We assume also that a circular buffer of size $2 *$ Buffer_size $e_{i}$ is reserved in the buffer cache of node $\wp_{i}$. In each period, while the presentation is consuming Buffer_size $e_{i}$ bits of data from its buffer, the other Buffer_size $e_{i}$ bits that the presentation will consume in the next period are retrieved from the storage servers into the buffer. This ensures that each presentation will have sufficient data to display the corresponding streams continuously. In addition, we define the display rate for each task $\tau_{i}$ (the rate at which data are consumed from the buffer for presentation purposes), using the equation:

$$
\begin{array}{r}
\text { display_rate }_{i}=\text { spatial_resolution }_{i} * \\
\text { temporal_resolution } \\
\text { tem }_{2} .
\end{array}
$$

Then, we require the following equalities to hold:

$$
\begin{aligned}
& C_{c s}^{i} * \text { Bandwidth }_{i}=\text { Buffer_size } \\
& \text { display_rate }_{i} * T_{i}=\text { Buffer_size }
\end{aligned}
$$

Equation 4 is used to evaluate the deterministic computation requirements within the critical sections of the tasks. Equation 5 states that with known display rate and buffer size for each task then the duration of consuming all the available data from the local buffer can be evaluated. This time duration represents the period $T_{i}$ of the task $\tau_{i}$. The value $C_{n o n_{-} c s}^{i}$ is the computation requirement of task $\tau_{i}$ to process the received data frames of size Buffer_size $e_{i}$.

In our example, the expected quality of every presentation and the resource requirements $R$ of every presentation 
Table 1. Parameters of task set in example

\begin{tabular}{|c|c|c|c|c|c|c|}
\hline \multicolumn{7}{|c|}{ Parameters of Task Set } \\
\hline Task & Spat. Res. & $f p s$ & $R$ & $T$ & $C_{\text {non-cs }}$ & $C_{\text {cs }}$ \\
\hline$\tau_{1}$ & $160 \times 120 \times 8$ & 22 & $r_{3}, r_{1}$ & 12 & 3 & 2 \\
\hline$\tau_{2}$ & $140 \times 110 \times 8$ & 20 & $r_{3}, r_{2}$ & 14 & 3 & 2 \\
\hline$\tau_{3}$ & $130 \times 110 \times 8$ & 19 & $r_{1}, r_{4}$ & 25 & 4 & 3 \\
\hline$\tau_{4}$ & $120 \times 100 \times 8$ & 15 & $r_{3}, r_{5}$ & 31 & 6 & 3 \\
\hline
\end{tabular}

of each task are listed in Table 1. The computed values of the periods and computation times within and outside critical sections for each task are listed in Table 1 in the corresponding columns. The worst-case blocking duration of each task is the sum given in formula 2 and it is determined as follows:

- Task $\tau_{1}$ :

$$
\begin{aligned}
& -H_{1}=\emptyset \quad-L_{1}=\left\{\tau_{2}, \tau_{3}, \tau_{4}\right\} \\
& -\beta_{1}=\max \left(C_{c s}^{2}, C_{c s}^{3}, C_{c s}^{4}\right)=3 \\
& -B_{1}=\beta_{1}+\sum_{\forall \tau_{i} \in H_{1}} \alpha_{i}^{1}=\beta_{1}=3 \text { since } H_{1}=\emptyset
\end{aligned}
$$

- Task $\tau_{2}$ :

$-H_{2}=\left\{\tau_{1}\right\} \quad-L_{2}=\left\{\tau_{4}\right\} \quad-\beta_{2}=C_{c s}^{4}=3$

$-B_{2}=\beta_{2}+\sum_{\forall \tau_{i} \in H_{2}} \alpha_{i}^{2}=\beta_{2}+\alpha_{1}^{2}=\beta_{2}+$

$\left(B_{1}+C_{c s}^{1}\right)=3+(3+2)=8$ and the condition $(3+2)<12$ holds (where 12 is the period of $\tau_{1} \in H_{2}$ ).

- Task $\tau_{3}$ :

$-H_{3}=\left\{\tau_{1}\right\} \quad-L_{3}=\emptyset \quad-\beta_{3}=0$

$-B_{3}=\beta_{3}+\sum_{\forall \tau_{i} \in H_{3}} \alpha_{i}^{3}=\beta_{3}+\alpha_{1}^{3}=\beta_{3}+\left(B_{1}+\right.$ $\left.C_{c s}^{1}\right)=0+(3+2)=5$.

- Task $\tau_{4}$ :

$-H_{4}=\left\{\tau_{1}, \tau_{2}\right\} \quad-L_{4}=\emptyset \quad-\beta_{4}=0$

$-B_{4}=\beta_{4}+\sum_{\forall \tau_{i} \in H_{4}} \alpha_{i}^{4}=\beta_{4}+\left(\alpha_{1}^{4}+\alpha_{2}^{4}\right)=$

$\beta_{4}+\left(0+\left(B_{2}+C_{c s}^{2}\right)\right)=0+(0+(8+3))=11$ since $11<12$.

We have to notice here that the term $\alpha_{1}^{4}$ is zero according to formula 3 since there exists a task, $\tau_{2}$, where $\tau_{2} \in H_{4}, R_{1} \cap R_{2} \neq \emptyset$ and $T_{1}<T_{2}$. This means that $\tau_{1}$ does not contribute directly to the total value of $B_{4}$ but only indirectly since the blocking time $B_{2}$ of task $\tau_{2}$ includes the occurence of task $\tau_{1}$. have:

Computing now the criterion given in Theorem 3.2 we

- $\mathrm{i}=1 \quad C_{1}+B_{1}=5+3=8 \leq 12 \quad\left(T_{1}=12\right)$

- $\mathrm{i}=2 \quad C_{2}+B_{2}=5+8=13 \leq 14 \quad\left(T_{2}=14\right)$

- $\mathrm{i}=3 \quad C_{3}+B_{3}=7+5=12 \leq 25 \quad\left(T_{3}=25\right)$

- $\mathrm{i}=4 \quad C_{4}+B_{4}=9+11=20 \leq 31 \quad\left(T_{4}=31\right)$

We therefore determine that the above set of tasks $\left\{\tau_{1}, \tau_{2}, \tau_{3}, \tau_{4}\right\}$ is schedulable.

\section{Conclusions}

In this paper, we have presented a scheduling mechanism with an integrated resource allocation model that is analyzable and understandable at a high level. Given a set of multimedia applications we know in advance if they will meet their deadlines or not. This scheduling strategy has been designed for multimedia applications that operate in a distributed computing environment where every multimedia task is allocated on a different node and can require the use of global resources. It is an approach for deterministic guarantees and provides predictable distributed multimedia applications.

It is also important to note that the proposed strategy can be easily implemented especially on a distributed system since only a message passing scheme is required to be supported by the underlying software.

\section{References}

[1] G.Coulson, G. Blair, P. Robin, and D. Shepherd. Supporting Continuous Media Applications in a Micro-Kernel Environment. In O. Spaniol, editor, Architectures and Protocols for High-Speed Networks. Kluwer Academic Publishers, 1994.

[2] M. B. Jones, D. Rosu, and M. Rosu. CPU Reservations and Time Constraints: Efficient, Predictable Scheduling of Independent Activities. In Proceedings of the 16th ACM Symposium on Operating Systems Principles, October 1997.

[3] C. Lee, R. Rajkumar, and C. Mercer. Experiences with Processor Reservation and Dynamic QOS in Real-Time Mach. In Proceedings of the Multimedia Japan 96, April 1996.

[4] I. Leslie, D. McAuley, R. Black, T. Roscoe, P. Barham, D. Evers, R. Fairbairns, and E. Hyden. The Design and Implementation of an Operating System to Support Distributed Multimedia Applications. IEEE Journal on Selected Areas in Communications, 14(7):1280-1297, September 1996.

[5] C. Mourlas and C. Halatsis. Task Synchronization for Distributed Real-Time Applications. In Proceedings of the Ninth Euromicro Workshop on Real-Time Systems, pages 184-190. IEEE Computer Society, 1997.

[6] K. Nahrstedt and J. M. Smith. The QoS Broker. IEEE Multimedia, 2(1):53-67, Spring 1995.

[7] J. Nieh and M. S. Lam. The Design, Implementation and Evaluation of SMART: A Scheduler for Multimedia Applications. In Proceedings of the 16th ACM Symposium on Operating Systems Principles, October 1997.

[8] R. Rajkumar, C. Lee, J. Lehoczky, and D. Siewiorek. A Resource Allocation Model for QoS Management. In Proceedings of the IEEE Real-Time Systems Symposium, December 1997.

[9] L. Sha, M. Klein, and J. Goodenough. Rate Monotonic Analysis for Real-Time Systems. In A. van Tilborg and G. Koob, editors, Foundations of Real-Time Computing: Scheduling and Resource Management, chapter 5, pages 129-155. Kluwer Academic Publishers, 1991. 